${ }^{1}$ Departamento

de Dermatología,

Facultad de Medicina, Universidad de Chile, Santiago, Chile.

${ }^{2}$ Interno de Medicina,

Facultad de Medicina, Universidad de Chile, Santiago, Chile.

${ }^{3}$ Unidad de Atención

y Control En Salud

Sexual (UNACESS) del Hospital San José, Santiago, Chile

${ }^{4}$ Bioquímico, Servicio de Dermatología, Hospital Clínico

Universidad de Chile, Santiago, Chile.

Trabajo no recibió financiamiento.

Los autores declaran no tener conflictos de interés.

Recibido el 14 de octubre de 2019, aceptado el 26 de diciembre de 2019 .

Correspondencia a: Hans Gubelin Alvarado.

Email: hans.gubelin@ ug.uchile.cl

Artículo de InVestigación

\title{
Caracterización de Sífilis en Embarazadas controladas en la unidad de Atención y Control en Salud Sexual del Hospital San José (HSJ), Santiago de Chile (2010-2016)
}

Nicolás Moya ${ }^{1}$, Hans Gubelin ${ }^{2}$, Ester Santander ${ }^{3}$, Claudia Correa ${ }^{3}$, Paola Arévalo ${ }^{3}$, Viviana Zemelman ${ }^{4}$

\section{RESUMEN}

Introducción: La sífilis gestacional continúa siendo un problema de salud pública en el mundo. Produce severos efectos adversos en la madre y en el feto de no ser tratada. En Chile, el Ministerio de Salud ha establecido un tamizaje para esta infección cada 3 meses en el embarazo y al momento del parto. Un tratamiento adecuado y oportuno es capaz de prevenir todos los efectos adversos de la sífilis en el embarazo.

Métodos: Este fue un estudio transversal retrospectivo que incluyó a 406 embarazadas controladas en la Unidad de Atención y Control en Salud Sexual (UNACESS) del Hospital San José (HSJ) entre los años 2010-2016.

Resultados: Los resultados del estudio fueron que un $87,7 \%$ de las embarazadas eran chilenas y un $12,3 \%$ de otras nacionalidades. Las nacionalidades más frecuentes para el grupo de extranjeras fueron: $54 \%$ peruanas y $18 \%$ haitianas. $\mathrm{Al}$ ingreso al estudio, $47,5 \%$ de las embarazadas se encontraban en el segundo trimestre de embarazo. De todas las participantes, un 38,7\% se encontraba en riesgo de sífilis congénita.

Discusión: En total, un 23\% de las participantes presentó un falso positivo biológico, cifra similar a la reportada en otros estudios. En este estudio encontramos que, en gestantes extranjeras, había mayor proporción de diagnóstico tardío en que chilenas. Esto podría deberse a dificultades para ingresar a la atención en salud.

Conclusión: Pese a que en Chile contamos con buenas tasas de diagnóstico, el manejo de la sífilis gestacional podría ser mejorado con una detección y tratamiento temprano. Las extranjeras buscaron atención en salud más tarde que las chilenas, por lo tanto, recibieron tratamiento más tardío y con mayor riesgo de sífilis congénita. Esto se puede explicar por dificultades para ingresar al sistema de salud.

Palabras claves: Sífilis; Sífilis congénita; Embarazo; Chile; Inmigrantes.

\section{SUMMARY}

Introduction: Syphilis in pregnancy remains a global public health problem with severe outcomes if it is not treated properly. The Chilean Ministry of Health has established syphilis screening at three times during pregnancy, with a final retest is during labor. An adequate treatment can prevent all side effects of syphilis in pregnancy.

Methods: This was a descriptive, transversal study which included 406 pregnant women who consulted for potential syphilis at the Control and Treatment of Sexual Health Unit (UNACESS in Spanish) of San José Hospital (HSJ) in Santiago, Chile from 2010 to 2016.

Results: A 87,7\% of the pregnant women were Chilean, while $12,3 \%$ had a different nationality. Among immigrants, the most frequent nationalities were: peruvian 54\% and Haitian 18\%. At enrolment, $47,5 \%$ of the pregnant women were in their second trimester. $38,4 \%$ was at risk of congenital syphilis.

Discussion: Overall, 23,1\% of the participants had a false positive test, which is congruent with previously reported data. In this study, we found a higher rate of late diagnosis, mainly in the immigrant pregnant women, which could be due to difficulties in accessing healthcare and cultural matters.

Conclusion: Despite a high overall treatment rate, antenatal syphilis management in this population could be improved by earlier detection and treatment. Immigrant women sought attention later in pregnancy, thus receiving delayed treatment with higher risk of congenital syphilis. This could be explained by obstacles in their access to healthcare.

Key words: Syphilis; Congenital Syphilis; Pregnancy; Chile; Immigrants. 
$\mathrm{L}$ a sífilis es una infección de transmisión sexual causada el Treponema pallidum. Continúa siendo la infección congénita más frecuente y puede producir efectos adversos materno-fetales severos. Más de un millón de mujeres embarazadas en el mundo se contagia cada año y más de la mitad de estas mujeres al no recibir tratamiento, presenta un resultado perinatal adverso como aborto, muerte fetal, parto prematuro, bajo peso al nacimiento y secuelas de sífilis en el recién nacido. ${ }^{1}$

En el año 2016, en Estados Unidos, se observaron las tasas más altas de sífilis congénita llegando a 15,7 casos por 100.000 nacidos vivos. ${ }^{2}$ En Chile la tasa de incidencia sífilis gestacional es de 35,5 casos por 100.000 habitantes gestantes. ${ }^{3}$ En américa latina, se estima una prevalencia global de sífilis gestacional de un $2.6 \%$, siendo en Brasil de $1.6 \%$, en y en Bolivia de un $4.3 \% .^{4-6}$ En Chile, el tamizaje de sífilis en embarazadas es norma desde 1976 y actualmente se encuentra en el marco de la Ley de Garantías Explícitas en Salud (GES). Este tamizaje se realiza mediante la prueba no treponémica como el VDRL (Venereal Disease Research Laboratory) o RPR (Ragina plasmática rápida), la cual se toma al ingreso del control perinatal, en el segundo control a las 24 semanas, en el tercer control las 3234 semanas de gestación y al momento del parto inmediato. Toda prueba no treponémica reactiva debe ser cuantificada. La confirmación se realiza con una prueba treponémica, utilizándose en Chile

la MHA-Tp (Microhemaglutination Assay for Antibody to Treponema Pallidum) que se procesa en centros certificados por el Instituto de Salud Pública de Chile (ISP). El diagnóstico y tratamiento oportuno y adecuado de la gestante y de las parejas sexuales, permite prevenir en un $100 \%$ la enfermedad del recién nacido, debiendo realizarse un adecuado seguimiento al neonato en el tiempo cuando la madre no fue tratada, si no recibió tratamiento de forma adecuada, o si hay sospecha clínica o de laboratorio sugerente. ${ }^{7}$ En este estudio, se pretendió conocer la problemática de la sífilis gestacional en un centro de derivación especializado en el diagnóstico y tratamiento de esta enfermedad en el área norte de Santiago.

\section{Овjetivo}

Describir las características clínicas y demográficas más relevantes de las embarazadas con sífilis, controladas en la UNACESS del Hospital San José de Santiago de Chile entre enero del 2010 y diciembre del 2016.

\section{MATERIALES Y MÉTODOS}

Estudio descriptivo transversal, en que los datos se obtuvieron de las fichas clínicas de embarazadas que consultaban en la Unidad de Atención y Control en Salud Sexual (UNACESS) del Hospital San José (HSJ), entre enero del 2010 y diciembre del 2016, por sospecha de sífilis gestacional, definida por un examen de VDRL reactivo a cualquier dilución.

Este estudio fue aprobado por el Comité de ética del Servicio de Salud Metropolitano Norte. Debido a que es un estudio retrospectivo, no se obtuvieron consentimientos informados.

Griterios de Inclusión: Mujeres gestantes derivadas desde algún centro de salud público o privado o que consultaron de forma espontánea en la UNACESS del HSJ por sospecha de sífilis gestacional confirmada por MHA-Tp.

Griterios de exclusión: Mujeres gestantes derivadas desde algún centro de salud público o privado o que consultaron de forma espontánea en la UNACESS del HSJ por sospecha de sífilis gestacional y que se descarta el diagnóstico mediante MHA-Tp.

Los datos fueron recopilados en una planilla de cálculo con la versión 16.0 Excel para MAC (Microsoft Corporation, Redmond, Washington, EE.UU) creada para esta finalidad, en donde se codificó la identidad de los pacientes para resguardar su identidad, siendo esta solo conocida por los autores en dicha unidad. El análisis de 
los datos se realizó con la versión 13.1 de Stata (StataCorp, College Station, TX, EE. UU.). El análisis de datos se realizó con estadísticas descriptivas simples como promedio, medias, desviación estándar (DE) y test estadístico para comparación de medias para la comparación de variables.

\section{Resultados}

Durante el período establecido entre el 1 de enero del 2010 y el 31 de diciembre del 2016, fueron derivadas a la UNACESS por sospecha de sífilis gestacional, 528 mujeres gestantes que presentaron un examen VDRL reactivo a cualquier dilución, el 99,5\% provenía desde Atención Primaria de Salud (APS) del área norte de Santiago y un $0,5 \%$ desde otros lugares (consultas privadas y otros servicios médicos hospitalarios del HSJ), no existiendo consultas espontáneas durante el periodo. Al llegar a la UNACESS del HSJ, a todas las gestantes se les realizó la prueba MHA-Tp con el fin de confirmar o descartar el motivo de derivación, determinándose que un 23,1\% de estas fueron falsos positivos, definidos por VDRL reactivo a cualquier dilución y MHA-Tp no reactiva. En estas pacientes se descartó el diagnóstico y se les derivó a Atención Primaria de Salud (APS) para estudio. Finalmente, durante el período estudiado ingresaron a control 406 gestantes, presentando un promedio de 58 ingresos de gestantes por año, cifra que se mantuvo estable exceptuando el año 2014 en donde aumento la cifra a 79 ingresos.

En relación con la nacionalidad de las 406 gestantes, 356 fueron chilenas, lo que corresponde al 87,7\% y 50 fueron extranjeras, lo que corresponde al 12,3\%. En este último grupo, el 54\% provenía de Perú, 18\% de Haití, 14\% de Colombia, 4\% de República Dominicana, 4\% de Bolivia, $4 \%$ de Paraguay y 2\% de Argentina (Grafico 1). Es importante destacar que el año 2010 solo ingresaron gestantes chilenas y que a partir del año 2011 ingresaron gestantes extranjeras (Grafico 2), inicialmente solo de origen peruano, para luego desde el año 2012 sumarse otras nacionalidades como la haitiana, constituyendo el segundo grupo en control en la unidad, seguido por las colombianas. El promedio de edad al momento de ingreso fue de 27 años: en gestantes chilenas fue de 26 y en extranjeras de 30 años. La mediana de edad fue de 26 años, siendo en las chilenas 25 años, lo que es significativamente menor en comparación con las extranjeras, con mediana de 29 años $(\mathrm{P}<0.01)$ (Tabla 1). El nivel de

\section{Gráfico 1}

Porcentaje de gestantes inmigrantes diagnosticadas de sífilis según nacionalidad (2010-2016)

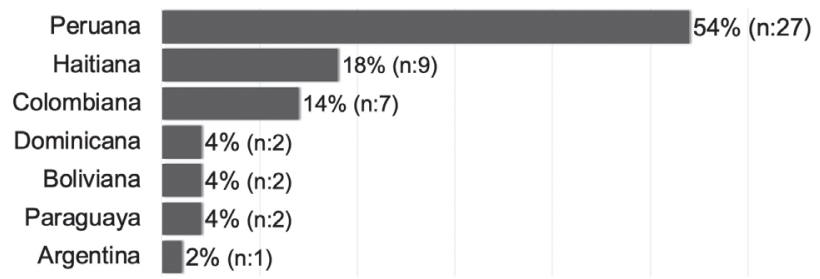

$n=50$

\section{Gráfico 2}

Porcentaje de gestantes inmigrantes diagnosticadas de sífilis chilenas y extranjeras según año de ingreso (2010-2016)

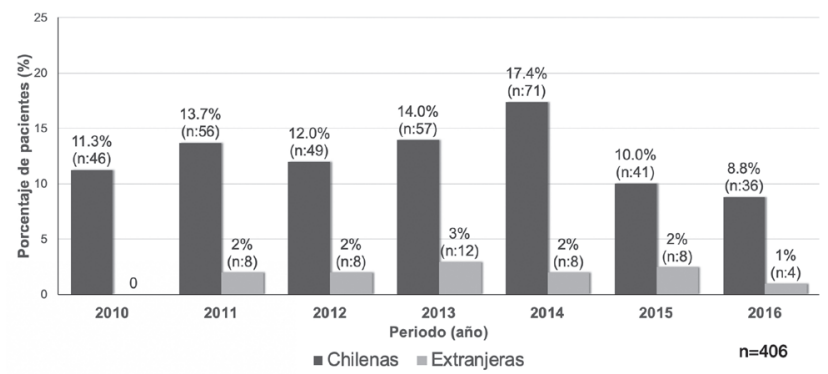

Tabla 1

Caracterización etaria en chilenas y extranjeras

\begin{tabular}{c|c|c|c|}
\hline & $\begin{array}{c}\text { Total de } \\
\text { gestantes }\end{array}$ & $\begin{array}{c}\text { Edad Min/Max } \\
\text { (años) }\end{array}$ & $\begin{array}{c}\text { Mediana de } \\
\text { edad (años) }\end{array}$ \\
\hline Chilenas & 356 & $15-42$ & $25^{*}$ \\
\hline Extranjeras & 50 & $17-43$ & $29^{*}$ \\
\hline Total & 406 & $15-43$ & 26 \\
\hline
\end{tabular}

\section{Gráfico 3}

Porcentaje de gestantes diagnosticadas con sífilis según trimestre del embarazo al momento del ingreso (2010-2016)

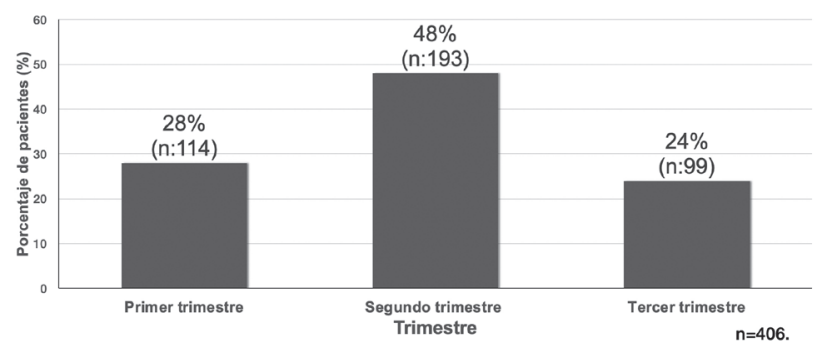




\section{Gráfico 4}

Porcentaje de gestantes chilena y extranjeras diagnosticadas con sífilis según trimestre del embarazo al momento del ingreso (2010-2016)

Chilenas

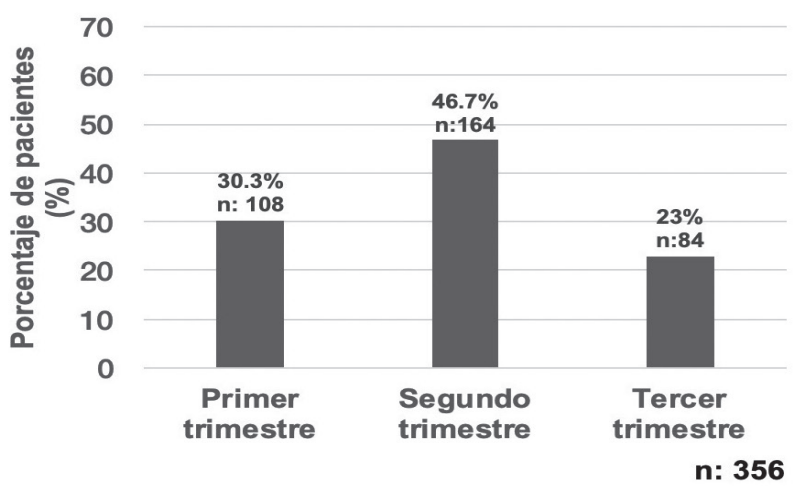

Gráfico 5

Porcentaje de gestantes con sífilis chilenas y extranjeras según etapa de la enfermedad (2010-2016)

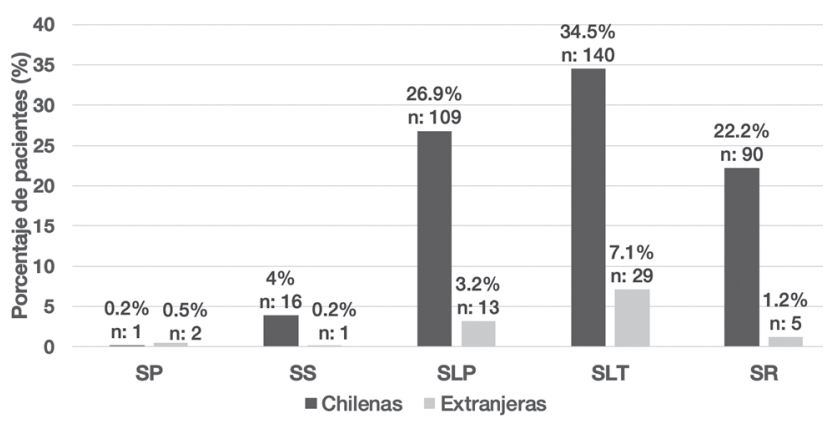

escolaridad de las gestantes fue de educación básica y media completa en el $65 \%$, seguido por solo básica en un $20 \%$ y universitaria solo en el $3 \%$, en el restante $12 \%$ no se contaba con este dato. Al momento del ingreso a la UNACESS, el 28\% de las gestantes cursaba el primer trimestre, el $48 \%$ el segundo y el $24 \%$ el tercer trimestre de embarazo (Gráfico 3). En relación a las gestantes chilenas, el 30\% ingresó durante el primer trimestre, el 46\% en el segundo y el $24 \%$ en el tercero, mientras que, en el grupo de las extranjeras, el 12\% en el primero, el 58\% en el segundo y el 30\% en el tercer trimestre (Gráfico 4).

Respecto a la etapa evolutiva de la sífilis, en ambos grupos, un $0.7 \%$ fue primaria y un $4.2 \%$ secundaria, catalogándose a un 30\% como latente precoz (SLP), a un $41,6 \%$ como latente tardía (SLT) y a un $23.4 \%$ como serología residual (SR), cada una de ellas definida según la Norma de Profilaxis, Diagnóstico y Tratamiento de las Infecciones de Transmisión Sexual (ITS) 2016. ${ }^{8}$ También se compararon estas etapas de la sífilis en chilenas
Extranjeras

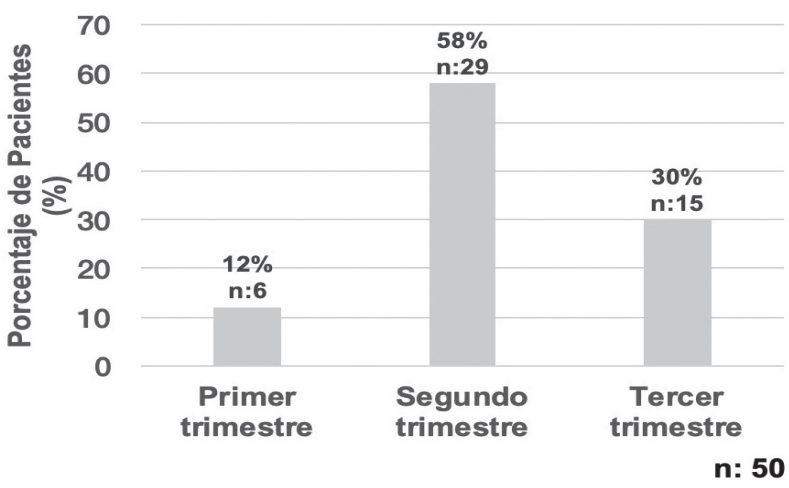

y extranjeras (Gráfico 5). En cuanto a sus comorbilidades, 2 gestantes correspondían a personas viviendo con VIH (una chilena y otra peruana), estando solo una de ellas en control en inmunología. En relación con otros antecedentes relevantes de las consultantes chilenas, podemos señalar que un 26,4\% tenía antecedentes de sífilis, un 5,3\% tenía mal control prenatal, definido por ingreso tardío o inasistencia a los controles, un $4,2 \%$ con antecedentes de policonsumo de drogas (en su mayoría pasta base y marihuana) y un $0,6 \%$ presento condilomatosis genital al momento del ingreso. De las pacientes extranjeras, solo una gestante de origen peruana refirió antecedentes de sífilis anterior tratada. Respecto al tratamiento, se utilizó penicilina benzatina intramuscular en un $95,3 \%$ de las pacientes y eritromicina en un $4,68 \%$, debido a antecedentes de alergia a la penicilina entregados por la gestante, dato obtenido desde la ficha clínica o auto referido por la gestante, esto corresponde a un $4,5 \%$ de las gestantes chilenas, un 3,7\% de las gestantes peruanas y un $28,6 \%$ de las colombianas. A todas se les controló con VDRL mensual hasta el momento del parto, no contando con el resultado de este último examen. En cuanto al riesgo de sífilis congénita, se consideraron en riesgo a aquellas embarazadas con etapas tempranas de la enfermedad, las cuales son sífilis primaria, sífilis secundaria y sífilis latente precoz y a las que no recibieron un adecuado esquema de tratamiento con penicilina para la etapa de la enfermedad correspondiente. ${ }^{8,9}$ El 38,4\% de todas las gestantes se clasificó con esta condición y $61,6 \%$ se estimó sin riesgo. En cuanto al riesgo fetal un $38,2 \%$ de las chilenas y a un $42 \%$ de las extranjeras se clasificó en esta condición, siendo un 55.5\% haitianas, un $42,8 \%$ colombianas y $40,7 \%$ peruanas, constituyendo estos tres grupos la mayor cantidad de extranjeros. 


\section{Discusión}

En nuestro estudio, si bien el reporte de casos por año fue similar, en gestantes chilenas en el 2014, se presentó un aumento del 39\% sobre el promedio; mientras que en el 2013, en gestantes extranjeras se produjo un crecimiento del $44 \%$, alza concordante con la elevación de casos a nivel nacional entre 2010 y 2015 y con reportes internacionales que hablan de una incremento en la prevalencia de sífilis en gestantes en otras partes del mundo, especialmente durante el periodo 2009 y 2013. ${ }^{7,2}$

La gran mayoría de las gestantes fue de origen chileno, pero existe un grupo no menor de extranjeras que presentaron características especiales a partir del 2011, siendo la población peruana la más frecuente con un $54 \%$, seguido por la haitiana con un $18 \%$ y colombiana con un $14 \%$. Estas cifras son similares a los datos de la población inmigrante en Chile, en la cual, los grupos más importantes son los peruanos con $25 \%$ y los colombianos con $13,6 \%$ del total. ${ }^{10}$ Interesante es señalar que los haitianos correspondían al 3,4\% de la población migrante, pero fueron la segunda nacionalidad con mayor frecuencia de sífilis gestacional en nuestro estudio, no sólo esto, sino que también, presentaron diagnósticos más tardíos, lo que permite concluir que este grupo podría ser considerado de mayor riesgo. ${ }^{10}$ Este fenómeno podría explicarse por varias razones, como distinto idioma, menor accesibilidad al sistema de salud, culturales, entre otras.

Las extranjeras, en comparación con la población chilena, presentaron mayor edad promedio al momento del ingreso al programa. En nuestro estudio, tanto en el grupo de extranjeras, como en el de chilenas, el ingreso fue mayor en el segundo trimestre, solo el 12\% de las extranjeras ingresaron durante el primer trimestre versus el 30\% de las chilenas. Esto reflejaría un ingreso más tardío en la población extranjera, un ejemplo de esto es la población haitiana, en la cual, el 100\% ingresó durante el segundo y tercer trimestre. Esto es importante debido a que son el el segundo grupo de inmigrantes más frecuente en nuestra muestra antecedidos por la población peruana. ${ }^{11}$ Estos datos podrían explicarse por una menor afiliación a un sistema previsional por parte de la población inmigrante, con un 15,8\% sin afiliación, comparado con un $2.2 \%$ de la población nacional. ${ }^{10}$ Esto pone en evidencia un problema de acceso a salud, especialmente en la salud sexual y reproductiva de este grupo. El flujo de inmigrantes a Chile ha aumentado en los últimos años, en el año 2002, según el CENSO, solo representaban a un 1\% de la población y el 2016 correspondían a un $4 \% .{ }^{10}$ La inmigración es un tema relevante a nivel nacional, en estudio se evidenció un ingreso más tardío al control en salud por parte de las migrantes, sería interesante estudiar las causas de este hallazgo y las posibles barreras para el ingreso a la atención en salud de la población migrante.

Los médicos especialistas señalaron como principal diagnóstico en las fichas clínicas revisadas a la SLT, seguido por SLP, en ambas poblaciones, concordando con lo señalado en la literatura internacional. ${ }^{12}$ Esto es relevante, ya que al ser menos frecuentes los casos con etapas tempranas de la infección, existiría un menor riesgo de transmisión vertical. Solo un 4,9\% se encontraba en sífilis primaria o secundaria, siendo este el grupo de mayor riesgo de transmisión de la sífilis al feto. Por otro lado, la mayoría de las gestantes fueron diagnosticadas durante el segundo trimestre y la etapa de sífilis más frecuente fue de SLT, esto podría evidenciar una falla en el control durante los primeros meses del embarazo.

\section{Conclusión}

De las mujeres embarazadas atendidas en la UNACESS del HSJ, la gran mayoría eran chilenas, pero un grupo no menor correspondía a inmigrantes. De este último grupo, se evidenció un diagnóstico más tardío en trimestres más avanzados del embarazo y en etapas de sífilis más tardías en comparación a las chilenas, siendo importante destacar al grupo de haitianas donde un 100\% se diagnosticó en el segundo trimestre. La migración es una realidad que Chile esta experimentando hace algunos años y la tendencia indica que va al aumento; por lo que es esencial que el sector salud pueda considerar a esta población como grupo de riesgo y les de mayores facilidades para acceder a la atención necesaria. Dentro de las limitaciones de este estudio, se debe destacar que representa la realidad de un solo centro en la Región Metropolitana, por lo que se requiere de mayor investigación para evidenciar la realidad nacional. 


\section{REFERENCIAS}

1. Rac M, Revell P, Eppes C. Syphilis during pregnancy: a preventable threat to maternal-fetal health. Am J Obstet Gynecol. 2017;216(4):352-63

2. Lin JS, Eder ML, Bean SI. Screening for syphilis infection in pregnant women updated evidence report and systematic review for the us preventive services task force. JAMA - J Am Med Assoc. 2018;320(9):918-25

3. Cáceres K. Situación epidemiológica de sífilis. Rev Chilena Infectología 2018; 35 (3): 284-296

4. Davey D, Shull H, Billings J, Wang D, Adachi K, Klausner J. C. Prevalence of Curable Sexually Transmitted Infections in Pregnant Women in Low- and Middle-Income Countries From 2010 to 2015:A Systematic Review .Sex Transm Dis. 2016;20(2):163-78

5. Soeiro CM de O, Miranda AE, Saraceni V, Santos MC dos, Talhari S, Ferreira LC de L. Syphilis in pregnancy and congenital syphilis in Amazonas State, Brazil: an evaluation using database linkage. Cad Saude Publica. 2014;30(4):715-23

6. Villazón N, Conde C, Juárez L, Uribe-Salas F. Evaluation of a rapid diagnostic test to assess the prevalence of maternal syphilis in Bolivia. Rev Med Chile. 2009;137(4):515-21

7. Cáceres K, Martínez R. Situación epidemiológica de sífilis (CIE 10: A50-A53.9). Chile, 2016. Rev Chil infectología. 2018;35(3):284-96

8. Norma de profilaxis, diagnóstico y tratamiento de las infecciones de transmisión sexual (ITS). Ministerio de Salud, Santiago, Chile, 2016.6

9. Walker GJA. Antibiotics for syphilis diagnosed during pregnancy. Cochrane Database of Systematic Reviews 2001, Issue 3. Art. No.: CD001143. DOI: 10.1002/14651858.CD001143

10. CASEN 2017. INMIGRANTES. Síntesis de resultados [Internet]. observatorio social. 201766 [cited 2019 May 5]. Available from: http://observatorio.ministeriodesarrollosocial.gob.cl/documentos/ CASEN_2013_Inmigrantes_01_marzo.pdf

11. Díaz Tolosa RI. Ingreso y permanencia de las personas migrantes en Chile: Compatibilidad de la normativa chilena con los estándares internacionales. Estud Const. 2016;14(1):179-220

12. Townsend CL, Francis K, Peckham CS, Tookey PA. Syphilis screening in pregnancy in the United Kingdom, 2010-2011: a national surveillance study. BJOG An Int J Obstet Gynaecol. 2017;124(1):79-86 